\title{
Quality of life in bladder cancer patients receiving medical oncological treatment; a systematic review of the literature
}

\author{
G. A. Taarnhøj ${ }^{1}$, C. Johansen ${ }^{1,2}$ and H. Pappot ${ }^{1 *}$ (D)
}

\begin{abstract}
Background: Previous quality of life (QoL) literature in bladder cancer $(\mathrm{BC})$ patients has focused on finding the preferred urinary diversion while little is known about the QoL of patients in medical oncological treatment (MOT). We performed a systematic review to assess the existing literature on QoL in patients with muscle-invasive BC (MIBC) undergoing MOT.

Methods: A systematic search of Pubmed and Embase was performed. Inclusion criteria were studies containing QoL data for patients undergoing chemo- and/or radiotherapy. We extracted all QoL scorings at different time intervals and on the six most prevalent domains: overall QoL, urinary, bowel sexual symptoms, pain and fatigue. The study was carried out according to PRISMA guidelines for systematic reviews and GRADE was used to rate the quality of evidence from the included studies.

Results: Of 208 papers reviewed, 21 papers were included. Twenty-one different QoL instruments were applied. The only data on QoL during chemotherapy was from patients in clinical trials investigating new treatments. No studies were found for patients in neoadjuvant treatment. The level of evidence at each time point was graded as very low to moderate. From the studies included the overall QoL seemed inversely related to the organspecific impairment from sexual and urinary symptoms and increased with decreasing organ-specific symptoms for long term survivors $>6$ months after treatment.

Conclusions: Collection of data on QoL from patients with MIBC disease undergoing MOT has been sparse and diverse. The present data can act as a summary but prompts for more prospective collection of QoL data from BC patients.
\end{abstract}

Keywords: Bladder cancer, Chemotherapy, Muscle-invasive bladder cancer, Quality of life, Urothelial cancer

\section{Background}

Despite recent literature highlighting the evident benefit of regular symptom reporting and early handling of side effects by patient-reported outcomes, the implementation of such in daily practice has yet to occur [1-3]. For patients receiving chemotherapy, clinical trials have traditionally informed us about quality of life (QoL) for these patients retrospectively as part of study reporting. These reports, however, inform us about highly selected patient populations eligible for enrollment in clinical trials, and

\footnotetext{
*Correspondence: helle.pappot@regionh.dk

${ }^{1}$ Department of Oncology, Rigshospitalet, Blegdamsvej 9, section 5073, 2100 Copenhagen $\varnothing$, Denmark

Full list of author information is available at the end of the article
}

are often not containing cancer specific modules as highlighted by three reviews in the field of bladder cancer published 1999-2005 [4-6]. Thus, our knowledge of QoL outside clinical trials remains sparse.

Bladder cancer $(\mathrm{BC})$ patients are characterized by heterogenic prognostics due to variation in their extent of disease as illustrated by the division into non-muscle invasive (NMIBC)/TaT1CIS, muscle invasive bladder cancer (MIBC)/T2-T4 and metastatic BC. Great interest and effort has been put into understanding health-related quality of life and symptomatic issues affecting the overall QoL for MIBC patients having undergone surgical procedures [7, 8]. Little is to the authors' knowledge

(c) The Author(s). 2019 Open Access This article is distributed under the terms of the Creative Commons Attribution 4.0 International License (http://creativecommons.org/licenses/by/4.0/), which permits unrestricted use, distribution, and reproduction in any medium, provided you give appropriate credit to the original author(s) and the source, provide a link to the Creative Commons license, and indicate if changes were made. The Creative Commons Public Domain Dedication waiver (http://creativecommons.org/publicdomain/zero/1.0/) applies to the data made available in this article, unless otherwise stated. 
known of the $\mathrm{BC}$ patients undergoing medical oncological treatment.

As part of the planning of a randomized patient-reported outcomes study in the $\mathrm{BC}$ population receiving medical oncological treatment, we set out to review the current literature for $\mathrm{BC}$ patients receiving chemotherapy. The aim of this study is to gather evidence of the QoL issues affecting the lives of $\mathrm{BC}$ patients during all phases of their disease, from diagnosis to treatment and thereafter thus informing us of potential gaps in the literature. The results will furthermore assist in determining which symptomatic patient-reported outcomes to be used in a coming randomized trial. We therefore present a systematic review of the QoL literature published on patients with locally advanced or metastatic BC undergoing chemotherapy.

\section{Methods}

\section{Search criteria}

A systematic search was performed in PubMed using the MeSH-terms 'quality of life, 'urinary bladder neoplasms,' 'drug therapy' and included 'quality of life, 'bladder cancer' and 'chemotherapy' as title or abstract terms (Additional file 1). The same search strategy was used in Embase. A professional, full-time librarian assisted the search to ensure systematics. The results were examined by title (author GAT) and if found relevant abstract and papers were read (GAT). Papers were found relevant if they included quantitative QoL data from patients with MIBC undergoing chemotherapy at any time point before, during or after their diagnosis or treatment. Radiotherapy as treatment modality was included because no papers with post-treatment QoL data were found for patients having undergone chemotherapy. To limit the search to the relevant population the following exclusion criteria were applied:

- not available in English

- published before 2000 thereby allowing for a slight overlap with the previous reviews in order not to dismiss valuable studies

- only comparing surgical procedures

- only involving NMIBC

- only abstract available.

Finally, to expand the results due to a modest number of relevant studies, cross-references in the included articles were examined. The search is graphically presented according to the PRISMA consort diagram (Fig. 1) and was carried out according to the PRISMA guidelines as a systematic review (Additional file 2) [9].

\section{Data extraction and analysis}

In order to construct graphs eliciting QoL over time, all QoL measurements from the included papers (Table 1) were noted. All scores on other scales than $0-100$ were converted to a $0-100$ scale. Overall QoL is displayed with values $0-100$, with increasing scores illustrating a better QoL, while the domain specific items are presented with a 0-100 scale, with an increasing score implicating more impairment, displayed in Table 2 as percentage impairment. The questionnaires with a reverse scaling of the domain specific items than described above (the FACT-BL, the EPIC and the domain-specific part of the SF-12), are scored on a Likert-like scale with increasing scores representing better QoL/fewer symptoms. These scorings were reverse transformed to represent percentage impairment for comparability with the other studies. The FACT-BL questionnaire scores comprise urinary, bowel and sexual symptom distress in one score, thereby disabling unique symptom scores for these items. If no subgrouping into urinary, sexual and bowel scores was specified for the studies applying the FACT-BL, the FACT-BL scores were thus used in Fig. 2 for the graphical presentation of urinary, bowel and sexual symptoms, even though the FACT-BL score then was used multiple times, thereby perhaps over- or underestimating the given symptom. No scores from patients having undergone primary surgical treatment (radical cystectomy) were included in the analysis, thereby allowing summarized scores without the known post-operative and instrumental issues known to affect QoL influencing the results of this review.

The GRADE criteria were applied for systematic review of the studies included [10]. GRADE is a system for assessing the quality of the evidence for the chosen outcome. The chosen outcome in this review was the determination of whether the sum of the studies included at each time point could act as reliable evidence for assessing QoL for the given population. Downgrading was considered based on either risk of bias, inconsistency, indirectness, imprecision or apparent publication bias. A risk of bias was assessed if the studies included e.g. reported different outcomes, if the outcomes from one study were diverging or low compliance in the study introduced selection bias. Inconsistency was determined if there was a large amount of clinical heterogeneity across the studies, participants or outcomes or if the methods applied were different across studies. Indirectness was determined if the population or outcomes differed from the population or outcomes of interest. Imprecision was established if the number of patients included in the rating of the outcome was too small to give a valid estimate of the outcomes. Publication bias was assessed by funnel plots looking at the pattern of study results [10]. Reasons for each downgrading assessment according to the categories above along with 


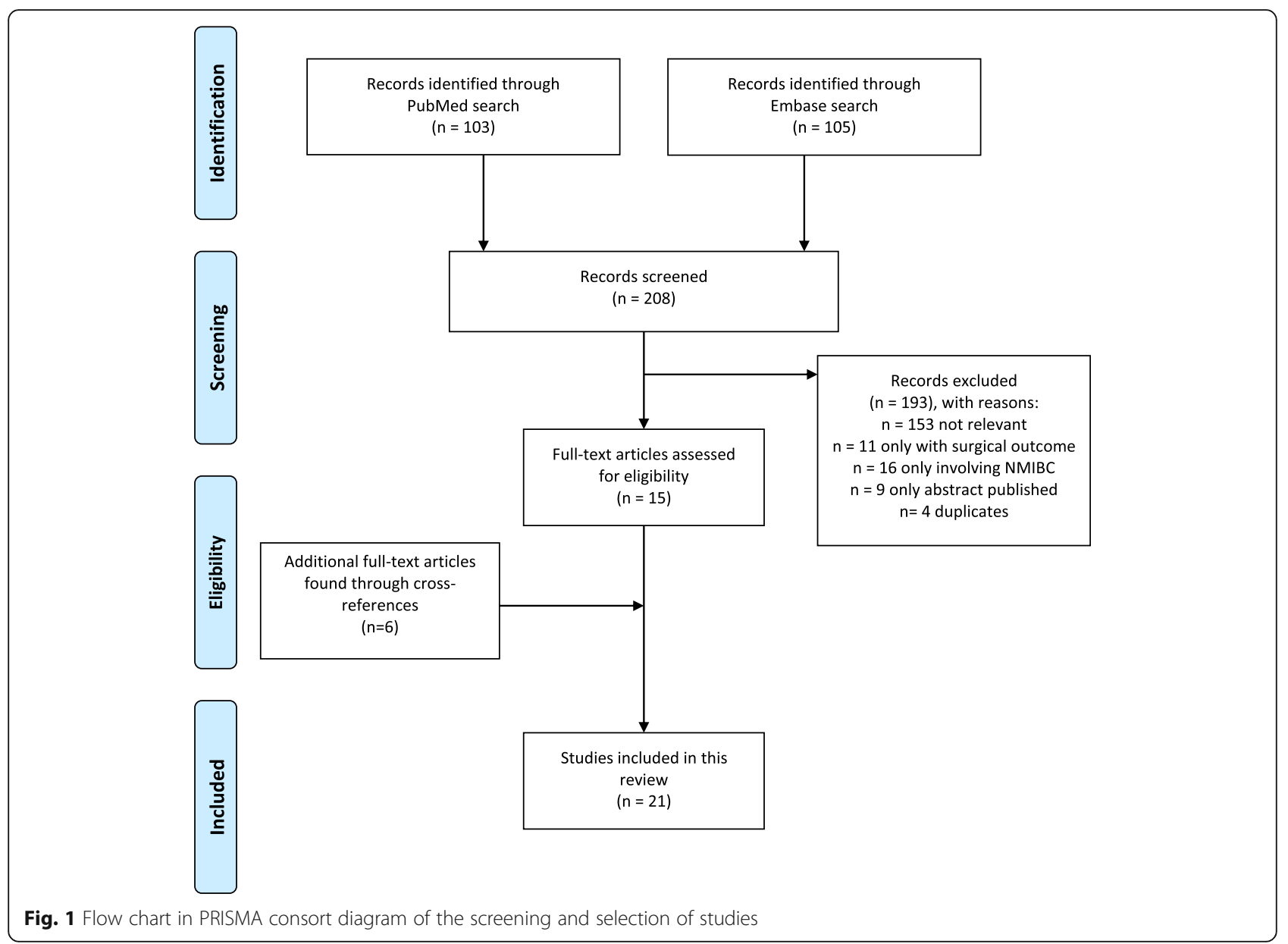

the findings of this analysis are given in Tables 3, 4, 5, 6 and the assessments reflect the degree of confidence we can have of the summarized QoL scores described above. The GRADE evaluation was done by two authors (GAT, HP).

\section{Ethical considerations}

This study did not require national or institutional approval.

\section{Results}

The search strategies in PubMed and Embase performed 2nd of July 2018, resulted in 103 and 105 scientific papers, respectively. All 208 titles were examined and if found relevant, abstracts and papers were read resulting in eight eligible papers from PubMed and eleven eligible paper from Embase. Four of these papers were overlapping. Thus fifteen papers were reviewed for the purpose of the study. Through cross-references a further six papers were included, resulting in a total of 21 eligible papers for review as illustrated in Fig. 1. The results are listed by topic in Table 1 .

\section{Quality of life instruments}

A total of 21 different QoL instruments were applied, most frequently the EORTC QLQ-C30, SF-36, FACT-G and FACT-BL, see Table 7, only displaying the validated instruments $(N=18)$. BC specific items were used in $52 \%$ of the included papers, FACT-BL being the most frequently used bladder specific measure. Five of the studies used non-validated questionnaires, some as a supplement to validated questionnaires, either developed by the investigator or modified from other validated questionnaires applied among other cancer groups, e.g., prostate cancer.

\section{Main topics}

All but two of the included studies defined the focus areas as opposed to letting the patient define the areas of most concern. The main focus areas listed in order of declining frequency were global QoL (17/21, 81\%), urinary symptoms $(12 / 21,57 \%)$, bowel symptoms (12/ $21,57 \%)$, sexual function $(10 / 21,48 \%)$, fatigue $(10 / 21$, $48 \%)$, pain $(8 / 21,38 \%)$ and anxiety/depression $(5 / 21$, $24 \%)$. For the above listed focus areas, a graphical presentation by disease phase is presented in Fig. 2 . 


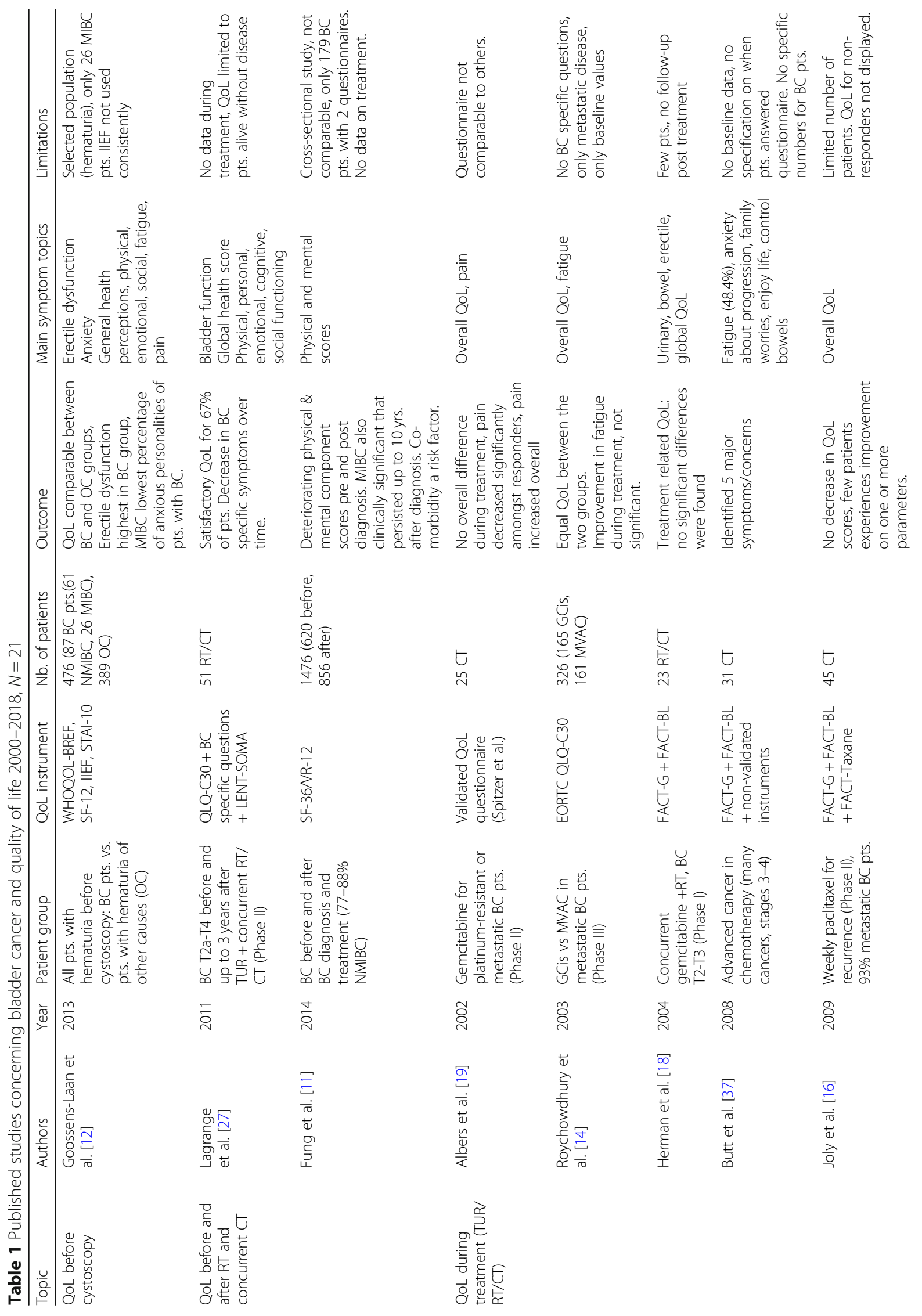




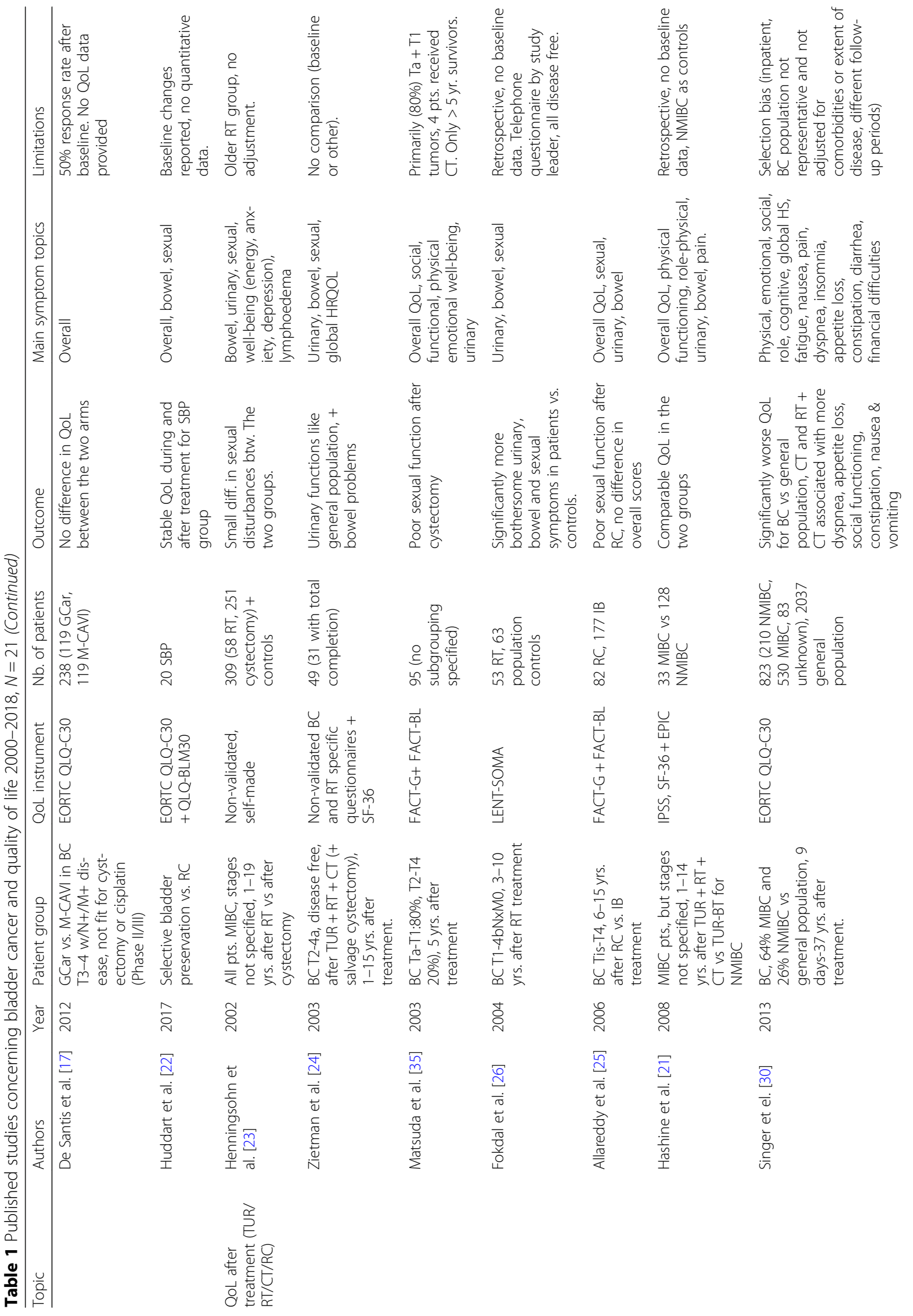




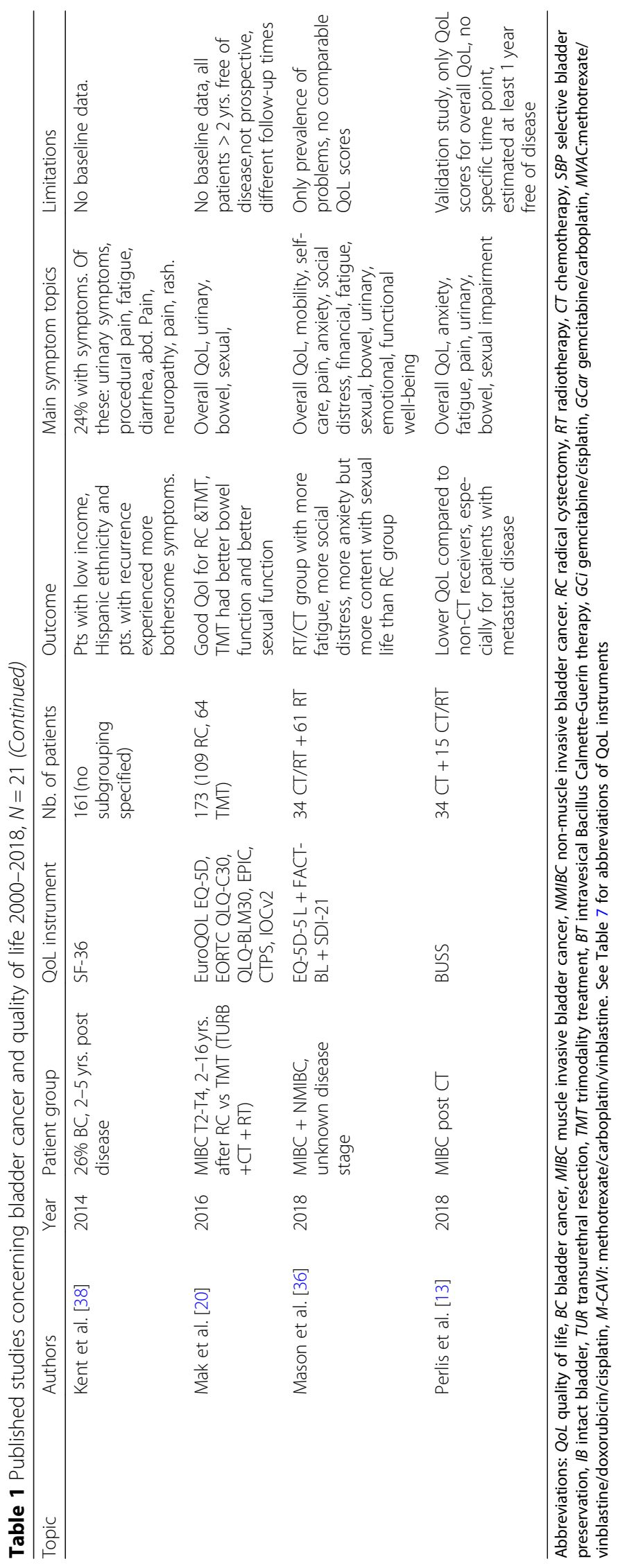




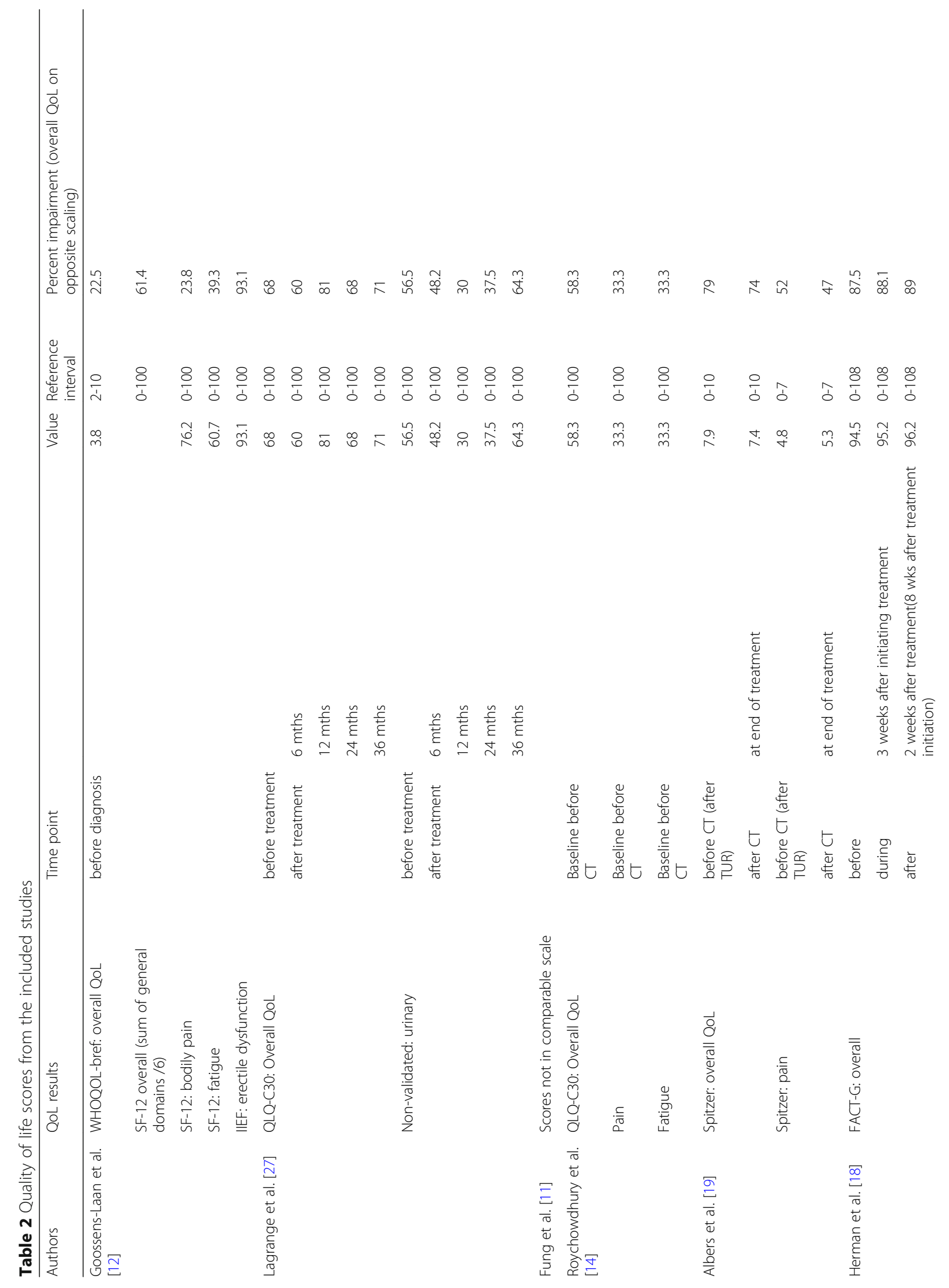




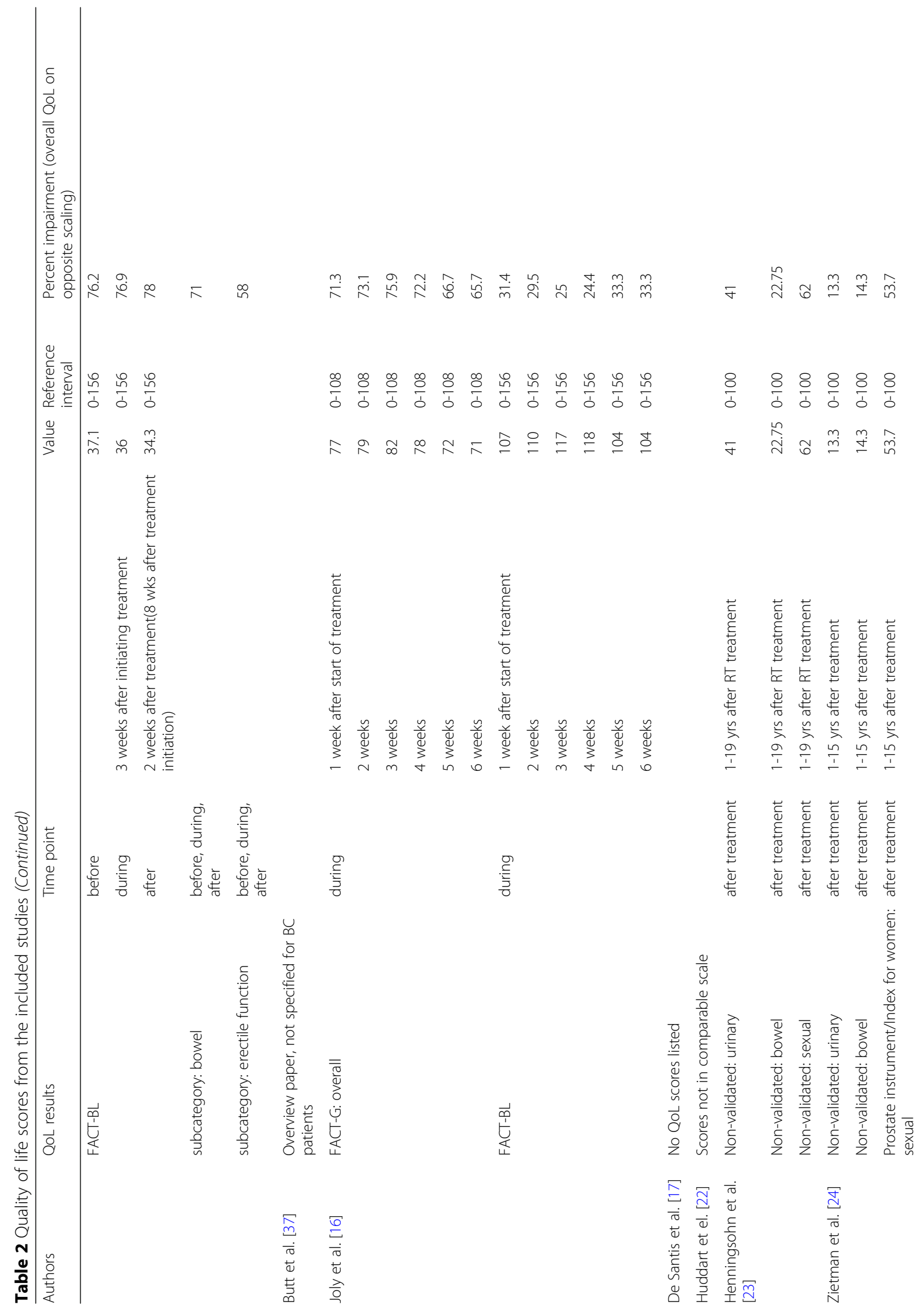




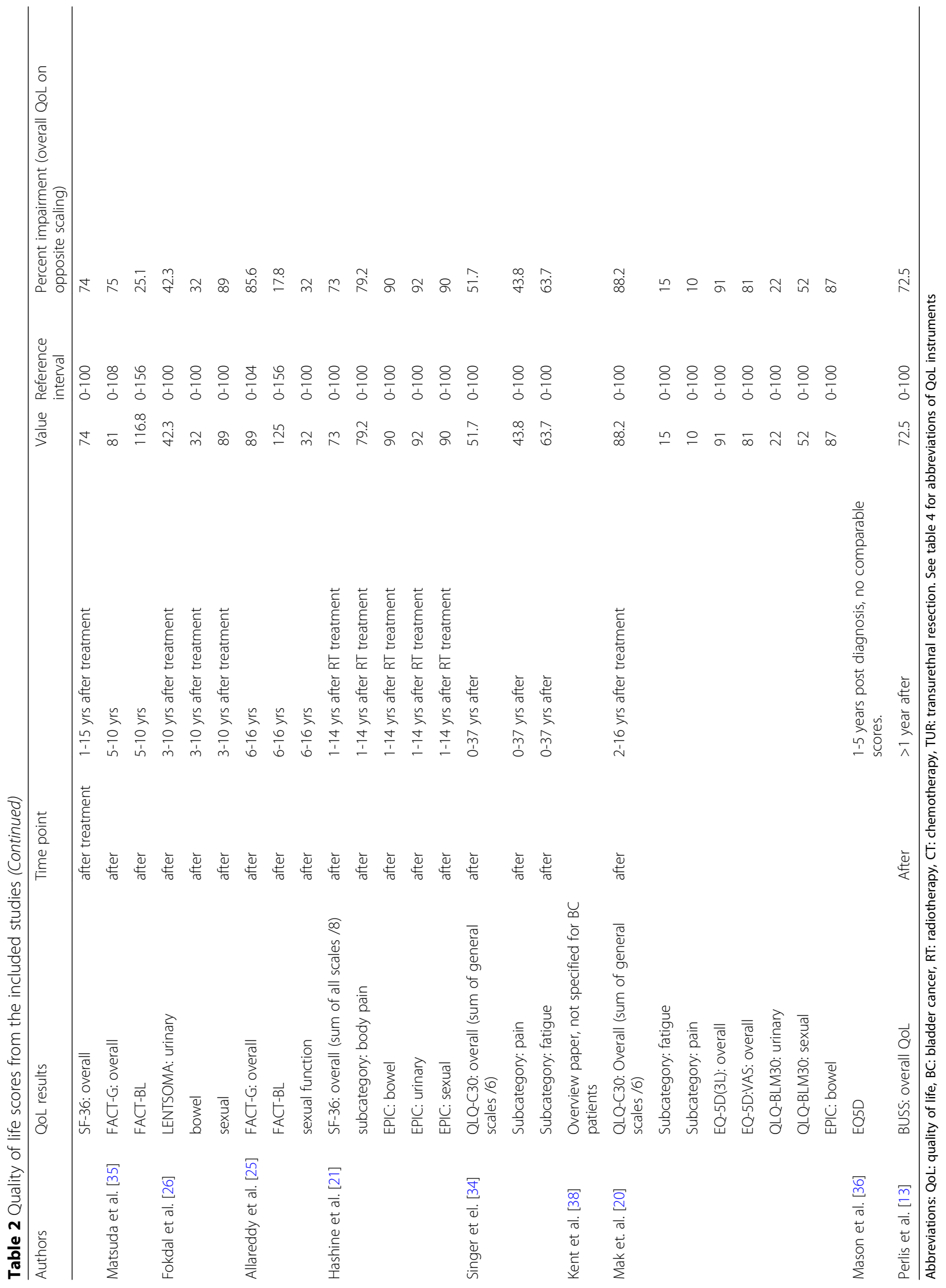



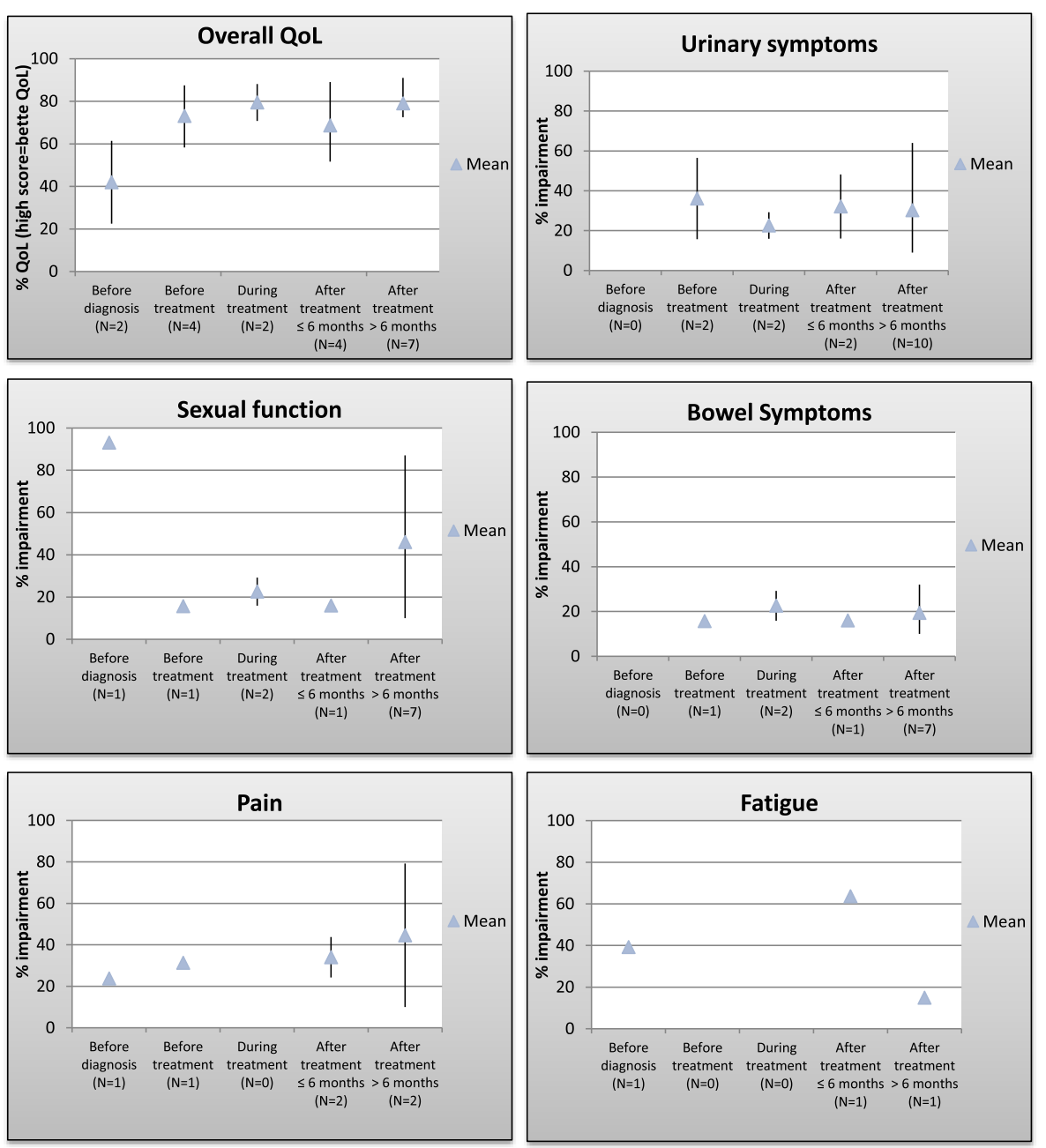

Fig. 2 Summarized quality of life scores during disease phases for bladder cancer patients

Anxiety/depression was not included in Fig. 2 because of a limited number of studies with this focus. Other focus areas were financial distress, nausea, dyspnea, insomnia, appetite loss, rash and neuropathy, but all were only listed once.

The studies reporting global QoL all had subdivided QoL into the following health related quality of life domains: physical, mental, social, cognitive, emotional and personal function.

\section{Patients}

Six of the 21 studies (29\%) presented data from only MIBC patients, whereas eight studies showed data from both the NMIBC and MIBC populations. Four studies presented QoL scores for patients with metastatic or recurrent disease, two studies presented data for a mix of MIBC and metastatic patients and one study reported data on patients defined by the authors as 'advanced' BC patients receiving chemotherapy, thereby potentially including both patients undergoing neoadjuvant, curative intended chemotherapy for locally advanced $\mathrm{BC}$ and metastatic $\mathrm{BC}$ patients.

\section{Treatment}

One study collected QoL data before cystoscopy. Thirteen of the 21 studies presented data from patients after receiving treatment, feasibly for comparison of two treatment modalities or to determine QoL for long-term survivors. Only two of these studies collected baseline data before treatment initiation. Seven studies measured QoL from patients undergoing chemotherapy, one of which was concurrent with radiotherapy in a Phase 1 trial, three presenting data from a Phase 2 trial and one from a Phase 3 trial. None of these studies had QoL as the primary outcome, and the Phase 1 trial was the only to include bladder cancer specific items. A total of six of the 21 studies did not list the QoL scores or were not on a 
Table 3 List of quality of life instruments applied, $N=18$

\begin{tabular}{|c|c|}
\hline $\begin{array}{l}\text { Abbreviated QoL } \\
\text { instrument }\end{array}$ & Full Title \\
\hline EORTC QLQ-C30 & $\begin{array}{l}\text { European Organization for Research and Treatment of Cancer Quality of life Core Questionnaire - for all cancer } \\
\text { patients }\end{array}$ \\
\hline EORTC QLQ-BLM30 & European Organization for Research and Treatment of Cancer Quality of life module for muscle invasive bladder cancer \\
\hline LENT-SOMA & Late Effects in Normal Tissue - Subjective, Objective, Management and Analytic scale for late effects of radiotherapy \\
\hline SF-36 & RAND Medical Outcomes Study Short Form 36 \\
\hline SF-12 & RAND Medical Outcomes Study Short Form-12 \\
\hline EPIC & Expanded Prostate Cancer Index Composite \\
\hline FACT-G & Functional Assessment of Cancer Therapy - General - for all cancer patients \\
\hline FACT-BL & Functional Assessment of Cancer Therapy - for patients with bladder cancer \\
\hline FACT-Taxane & Functional Assessment of Cancer Therapy - for patients receiving taxane therapy \\
\hline IPSS & International Prostate Symptom Score \\
\hline EuroQOL EQ-5D & EuroQOL Group non-disease specific QoL instrument \\
\hline CTPS & Cancer Treatment Perception Scale \\
\hline IOCv2 & Impact of Cancer version 2 \\
\hline HADS & Hospital Anxiety and Depression Scale \\
\hline WHOQOL-BREF & World Health Organization Quality of Life abbreviated version \\
\hline STAl-10 & State-Trait Anxiety Inventory-Trait scale short form \\
\hline IIEF & International Index of Erectile Function \\
\hline Spitzer index & Validated instrument for palliative patients \\
\hline
\end{tabular}

comparable scale and were therefore not included in the analyses. The treatments consisted of combinations of transurethral resection, radical cystectomy, partial cystectomy, ${ }^{1}$ electrocoagulation, nephrouretherectomy, installation of Balcillus Calmette-Guérin and radiotherapy with or without concurrent chemotherapy. There was a large disparity between the studies as one study had mainly surgically treated patients in stages Ta-T1 and very few patients in need of adjuvant treatment for more advanced stages while another study presented patients volunteering for an inpatient rehabilitation after their oncological treatment suggesting more invasive treatment and sequelae thereof.

\section{Quality of life outcome}

Two studies presented QoL before diagnosis as a baseline QoL not affected by the distress and change

Table 4 GRADE Summary of findings. Outcome: Urinary symptoms

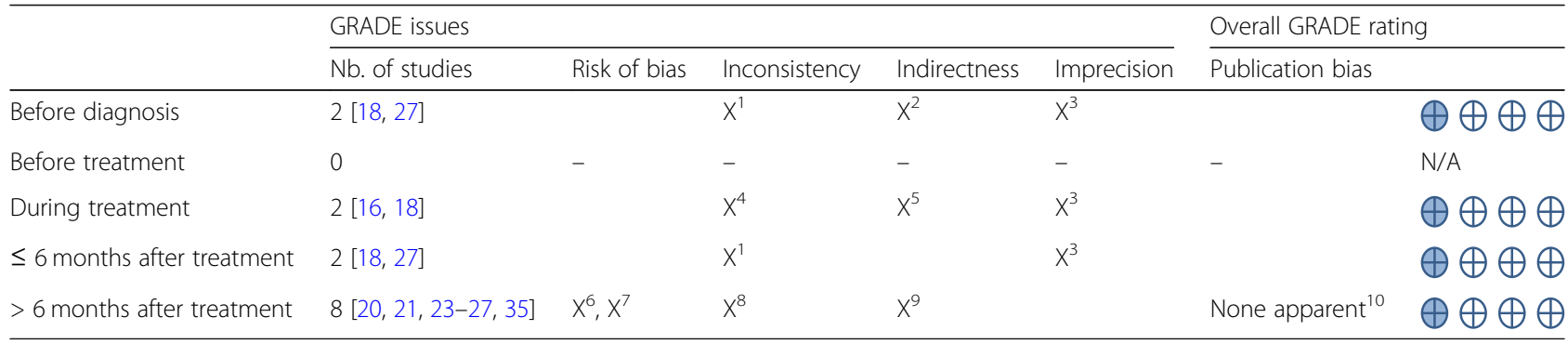

${ }^{1} 1$ out of 2 scores from non-validated questionnaire [27]

${ }^{2}$ No studies before CT for metastatic disease or as neoadjuvant treatment

${ }^{3}$ Large difference in populations hence diverging scores

${ }^{4}$ Small number of patients

${ }^{5}$ Only one study representing metastatic population, no studies representing neoadjuvant population

${ }^{6}$ Diverging scores from one study [27]

${ }^{7}$ Small number of patients for [27] at time point 36 months

${ }^{8}$ Different incl. Non-validated questionnaires used

${ }^{9}$ All patients after CT/RT or RT alone

${ }^{10}$ As estimated by forest plot 
Table 5 GRADE Summary of findings. Outcome: Sexual impairment

\begin{tabular}{|c|c|c|c|c|c|c|c|}
\hline & & GRADE issue & & & & & Overall GRADE rating \\
\hline & $\mathrm{Nb}$. of studies & Risk of bias & Inconsistency & Indirectness & Imprecision & Publication bias & \\
\hline Before diagnosis & $1[12]$ & $x^{1}, x^{2}$ & & & $x^{3}$ & & $\oplus \oplus \bigoplus \oplus$ \\
\hline Before treatment & $1[18]$ & $x^{1}$ & & & $x^{3}$ & & $\oplus \oplus \bigoplus \oplus$ \\
\hline During treatment & $2[16,18]$ & & $x^{4}$ & $x^{5}$ & $x^{3}$ & & $\oplus \oplus \oplus \oplus$ \\
\hline$\leq 6$ months after treatment & $1[18]$ & $X^{1}$ & & & $x^{3}$ & & $\oplus \oplus \oplus \oplus$ \\
\hline$>6$ months after treatment & $7[20,21,23-26,35]$ & & $x^{6}$ & & & None apparent $^{7}$ & $\oplus \oplus \oplus \oplus$ \\
\hline
\end{tabular}

${ }^{1}$ Only one study represented

${ }^{2}$ Selected population

${ }^{3}$ Small number of patients

${ }^{4}$ Large difference in populations hence diverging scores, despite same instrument used

${ }^{5}$ Only one study representing metastatic population, no studies representing neoadjuvant population

${ }^{6}$ Large range between scores despite comparable study populations, presumably due to different instruments used, incl. Non-validated instrument

${ }^{7}$ As estimated by funnel plo

of perspective of having a cancer diagnosis [11, 12]. Goossens-Laan et al. showed significantly poorer QoL scores on erectile and orgasmic function in the BC group vs. the group with hematuria from other causes while Fung et al. displayed overall QoL data with a significant fall in Physical and Mental Component Scores (PCS, MCS) from pre- to post-diagnosis, although results were not clinically meaningful with small relative differences in the two groups. For MIBC patients this fall in PCS remained significant and clinically meaningful for all times after diagnosis and was greatest for patients with multiple comorbidities. This latter finding was echoed by Perlis et al. for patients post treatment [13].

When looking at QoL during treatment, the by far largest study by von der Maase et al. (reported by Roychowdhury et al.) with a total of 326 patients in two treatment arms, reported improvement in QoL during the chemotherapy treatment for metastatic patients. However, the results were not found significantly different from baseline values and no bladder specific items were used [14, 15]. The studies by Joly, De Santis, Herman and Albers all presented stable overall QoL scores during chemotherapy treatment, although in the Albers study this was, as for pain values, only seen for responders [16-19]. Likewise, the study by Joly et al. found an improvement in QoL among 10\% of patients with objective response or stabilization of disease as a result of the treatment [16]. Herman et al. presented significantly lower bladder specific scores for patients receiving a higher dose of chemotherapy and lower overall QoL scores for those experiencing dose-limiting toxicities [18].

The QoL data from the after-treatment studies were collected from 0 to 37 years after $\mathrm{BC}$ diagnosis and treatment, rendering comparison somewhat difficult. However, for the patients having undergone radiotherapy as a bladder conserving strategy, the majority of the studies found the patients to have good or satisfactory bladder, bowel and/or sexual function and superior to that of cystectomy treated individuals when these were used as control groups [20-25]. Nonetheless, Fokdal et al. presented large impact on urinary, bowel and sexual function and reported high prevalence of disturbances; up to $94 \%$ impaired erective dysfunction while Lagrange et al. presented deteriorating over time. Comparison was

VTable 6 GRADE Summary of findings. Outcome: Bowel symptoms

\begin{tabular}{|c|c|c|c|c|c|c|c|}
\hline & \multirow[b]{2}{*}{$\mathrm{Nb}$. of studies } & \multicolumn{5}{|c|}{ GRADE issues } & \multirow[t]{2}{*}{ Overall GRADE rating } \\
\hline & & Risk of bias & Inconsistency & Indirectness & Imprecision & Publication bias & \\
\hline Before diagnosis & 0 & - & - & - & - & - & N/A \\
\hline Before treatment & $1[18]$ & $x^{1}$ & & & $x^{2}$ & & $\oplus \oplus \oplus \oplus$ \\
\hline During treatment & $2[16,18]$ & & $x^{3}$ & $x^{4}$ & $x^{2}$ & & $\oplus \oplus \oplus \oplus$ \\
\hline$\leq 6$ months after treatment & $1[18]$ & $X^{1}$ & & & $x^{2}$ & & $\oplus \oplus \oplus \oplus$ \\
\hline$>6$ months after treatment & $7[20,21,23-26,35]$ & & $x^{5}$ & & & None apparent ${ }^{6}$ & $\oplus \oplus \oplus \oplus$ \\
\hline
\end{tabular}

'Only one study represented

${ }^{2}$ Small number of patients

${ }^{3}$ Large difference in populations hence diverging scores, despite same instrument used

${ }^{4}$ Only one study representing metastatic population, no studies representing neoadjuvant population

${ }^{5}$ Large range between scores despite comparable study populations, presumably due to different instruments used, incl. Non-validated instruments

${ }^{6}$ As estimated by funnel plot 
Table 7 List of quality of life instruments applied, $N=18$

\begin{tabular}{ll}
\hline Abbreviated QoL instrument & Full Title \\
\hline EORTC QLQ-C30 & European Organization for Research and Treatment of Cancer Quality of life Core Questionnaire - for all cancer patients \\
EORTC QLQ-BLM30 & European Organization for Research and Treatment of Cancer Quality of life module for muscle invasive bladder cancer \\
LENT-SOMA & Late Effects in Normal Tissue - Subjective, Objective, Management and Analytic scale for late effects of radiotherapy \\
SF-36 & RAND Medical Outcomes Study Short Form 36 \\
SF-12 & RAND Medical Outcomes Study Short Form-12 \\
EPIC & Expanded Prostate Cancer Index Composite \\
FACT-G & Functional Assessment of Cancer Therapy - General - for all cancer patients \\
FACT-BL & Functional Assessment of Cancer Therapy - for patients with bladder cancer \\
FACT-Taxane & Functional Assessment of Cancer Therapy - for patients receiving taxane therapy \\
IPSS & International Prostate Symptom Score \\
EUroQOL EQ-5D & EuroQOL Group non-disease specific QoL instrument \\
CTPS & Cancer Treatment Perception Scale \\
IOCV2 & Impact of Cancer version 2 \\
HADS & Hospital Anxiety and Depression Scale \\
WHOQOL-BREF & World Health Organization Quality of Life abbreviated version \\
STAl-10 & State-Trait Anxiety Inventory-Trait scale short form \\
IIEF & International Index of Erectile Function \\
Spitzer index & Validated instrument for palliative patients \\
\hline
\end{tabular}

in the Fokdal study made with population controls while Lagrange presented data from only 6-7 individuals at 36 months [26, 27].

The QoL scores from the studies above are displayed in Table 2 and gathered graphically in Fig. 2 displaying the overall QoL and subdivisions into urinary, bowel and sexual symptoms as well as pain and fatigue over the time course of a MIBC patient's treatment. The GRADE evaluation was done for the overall QoL, urinary, sexual and bowel symptoms but not conducted for the outcomes fatigue and pain due to a very limited number of studies rendering GRADE analysis redundant.

Overall, we found that QoL has been immensely explored for MIBC patients post-treatment, free of disease, as shown by the GRADE analysis in Tables 3, 4, 5, 6. We found no studies reporting data during treatment for patients outside clinical trials, neither for the neoadjuvant nor metastatic population. From the summarized QoL scores in Fig. 2 it seems clear that especially urinary symptoms and sexual impairment are important issues for this group of patients. The GRADE analysis makes clear that Fig. 2 should be interpreted with caution due to the low level of evidence for almost all time-points.

\section{Discussion}

To the best of our knowledge, this review is the first to compile quality of life studies in $\mathrm{BC}$ patients receiving medical oncological treatment. We have portrayed a diversity in choice of QoL questionnaires and an absence of studies informing us about QoL in the neoadjuvant and metastatic populations outside clinical trials. The summarized QoL curves in Fig. 2 lead us to believe that urinary symptoms and sexual impairment impacts QoL substantially due to their inverse relationship over the course of time. The developmental curve of overall QoL illustrates a tendency of increasing QoL after initiating treatment followed by a fall in the early months after treatment. Subsequently QoL increases in survivors more than 6 months after treatment. Also, only few studies included psychological items in the QoL instruments or as a supplement. However, a previous study showed that bladder cancer diagnosis did not significantly affect the patients' levels of anxiety and depression [28], thus suggesting that QoL may not be significantly influenced by these issues in bladder cancer patients. Also, little attention has been paid to the psychosocial issues of the patients and the importance of these in relation to a person's QoL, which ultimately could explain the reported levels of QoL in the different domains [29]. These issues are described in the literature in the general cancer population [30-33]. From these reports it is not evident in which direction psychosocial difficulties interfere with a patient's QoL as the studies report diverging influences in the populations of interest, thereby rendering a need to understand how QoL is influenced by different psychosocial perspectives in the $\mathrm{BC}$ population.

Based on this review one may question whether there exists sufficient knowledge to reach the primary aim: to understand the QoL of $\mathrm{BC}$ patients undergoing medical 
oncological treatment. While the search string focused on patients in chemotherapy, radiotherapy studies were included because of the evident lack of post-treatment studies informing us about the QoL after chemotherapy treatment. The following apparent heterogeneity in content and design illustrated by the large variety of patients comprising either $\mathrm{BC}$ patients only with hematuria [12], recruited when in a clinical trial $[14-16,18,19]$, recruited in post-treatment clinic [34] or applying methodologically problematic study designs challenging the implications of results such as a cross-sectional setting [11], determining QoL for patients having undergone radiotherapy without applying radiotherapy-specific questionnaires $[20,21,23$, $26,34]$ or recruiting patients years after diagnosis with no baseline data $[20,21,23-26,35-38]$ renders careful conclusions about the development of QoL through a patient's phases of disease.

Having addressed these issues, the most apparent outlier in Fig. 2 deserves notice. The unmistakable and permanent decrease in sexual impairment from before cystoscopy to before treatment may be the parameter to best distinguish MIBC patients in medical oncological treatment from MIBC patients undergoing surgical treatment. The latter are described as having a substantial amount of sexual troubles after cystectomy, because of the accompanying prostatectomy, a development not described in this review $[39,40]$. Sexual problems are known to have a significant impact on QoL which makes this development in Fig. 2 a paramount finding in this review [41].

With the above issues in mind, this review is the first to gather the raw data from previous studies and create an overview of this diverse group of patients. Figure 2 may despite the apparent heterogeneity among the studies serve as the currently best guidance for physicians facing patients commencing medical oncological treatment. When discussing worries about QoL as a result of treatment, the results of this review may reassure patients unsure of future outcomes. Also, patients unsure of the effects of urological surgical interventions and searching for viable alternatives may need this platform of evidence to assist treatment decisions.

\section{Study limitations}

Our attempt to align different QoL instruments and further align various time points applied in different populations studied and assuming an equal weight of each study presents a clear limitation of the present review. The GRADE system, although systematic, does not take into account the large cultural differences between two otherwise comparable populations; a Japanese patient may score QoL higher than an American patient despite objectively the same burden of symptoms, or vice versa. This may be the reason for the variation in scores as listed in Table 2 and commented in Tables 3, 4, 5, 6 . Also, the combined scores of the FACT-BL instrument may both over- and underestimate the impairment in the urinary, bowel and sexual domains. The direction of this estimate cannot be determined. These issues constitute the need to interpret Fig. 2 with care, as illustrated by the GRADE analysis. Further, for some of the time points in this figure only one or two QoL scores make up the supposed overview of QoL development, perhaps falsely giving the single studies equal weight of time points comprising many studies. Unfortunately, this is a result of the scarce literature in this field and cannot be avoided. The scares literature in certain populations or time points may even give us valuable information of which patient groups have not been studied as intensively as others and guide us to future prospective research, in this case towards an understanding of the QoL for BC patients in neoadjuvant chemotherapy and for the metastatic population outside clinical trials. Lastly, although thorough literature search was performed in the two chosen databases including cross-references, the study group acknowledges that a number of studies describing QoL in advanced BC patients and specifically studies describing QoL during or after radiotherapy may, because of our chosen search string, not be included in our review. Also, given the nature of the QoL outcome and the psychological construct related to overall QoL, the results may have benefitted from a similar search in e.g. PsychInfo.

\section{Conclusions}

As set out to do, this review sheds new light on the issues at hand for MIBC patients before, during and after medical oncological treatment. It provides a listing of QoL issues important for BC patients to include in prospective patient-reported outcome trials and identifies a need for further efforts to describe the QoL issues with validated instruments for advanced and metastatic $\mathrm{BC}$ patients, especially during treatment.

\section{Endnote}

${ }^{1}$ From Matsuda et al. [35] without further explanation. May be cystectomy in women without resection of internal genitalia.

\section{Additional files}

Additional file 1: PubMed search string. (PNG $44 \mathrm{~kb}$ )

Additional file 2: PRISMA Checklist. (DOC 63 kb)

Acknowledgements

Not applicable. 


\section{Funding}

This work was supported by Danish Cancer Society [grant number R150A 10114]. The funding party played no role in any part of the design, conduction, analysis or publication plan for this study.

\section{Availability of data and materials}

All data generated or analysed during this study are included in this published article [and its Additional files].

\section{Authors' contributions}

Conception and design: GAT, CJ, HP. Data collection: GAT, HP. Data analysis: GAT, HP. Manuscript drafting: GAT, CJ, HP. All authors approved the final version of this manuscript.

\section{Ethics approval and consent to participate}

Not applicable.

\section{Consent for publication}

Not applicable.

\section{Competing interests}

The authors declare that they have no competing interests.

\section{Publisher's Note}

Springer Nature remains neutral with regard to jurisdictional claims in published maps and institutional affiliations.

\section{Author details}

${ }^{1}$ Department of Oncology, Rigshospitalet, Blegdamsvej 9, section 5073, 2100 Copenhagen $\varnothing$, Denmark. ${ }^{2}$ Unit of Survivorship, Danish Cancer Society, Strandboulevarden 49, 2100 Copenhagen $\varnothing$, Denmark.

Received: 23 July 2018 Accepted: 20 December 2018 Published online: 22 January 2019

\section{References}

1. Basch E, Deal AM, Kris MG, Scher HI, Hudis CA, Sabbatini P, et al. Symptom monitoring with patient-reported outcomes during routine Cancer treatment: a randomized controlled trial. J Clin Oncol. 2016;34(6):557-65.

2. Basch E, Deal AM, Dueck AC, Scher HI, Kris MG, Hudis C, et al. Overall survival results of a trial assessing patient-reported outcomes for symptom monitoring during routine Cancer treatment. JAMA. 2017;318(2):197-8.

3. Denis F, Lethrosne C, Pourel N, Molinier O, Pointreau Y, Domont J, et al. Randomized trial comparing a web-mediated follow-up with routine surveillance in lung Cancer patients. J Natl Cancer Inst. 2017;109(9):djx029djx044. https://academic.oup.com/jnci/article/109/9/djx029/3573360

4. Gerharz EW, Mansson A, Mansson W. Quality of life in patients with bladder cancer. Urol Oncol. 2005:23(3):201-7.

5. van der Veen $\mathrm{JH}$, van $\mathrm{AG}$, Kurth $\mathrm{KH}$. Quality-of-life assessment in bladder cancer. World J Urol. 1999;17(4):219-24.

6. Botteman MF, Pashos CL, Hauser RS, Laskin BL, Redaelli A. Quality of life aspects of bladder cancer: a review of the literature. Qual Life Res. 2003;12(6):675-88.

7. Ghosh A, Somani BK. Recent trends in Postcystectomy health-related quality of life (QoL) favors Neobladder diversion: systematic review of the literature. Urology. 2016;93:22-6.

8. Porter MP, Penson DF. Health related quality of life after radical cystectomy and urinary diversion for bladder cancer: a systematic review and critical analysis of the literature. J Urol. 2005;173(4):1318-22

9. Moher D, Liberati A, Tetzlaff J, Altman DG. Preferred reporting items for systematic reviews and meta-analyses: the PRISMA statement. PLoS Med. 2009;6(7):e1000097.

10. Ryan R, Hill, S. How to GRADE the quality of the evidence. Cochrane Consumers and Communication Group 2016 [Available from: https:// figshare.com/articles/How_to_GRADE/6818894]. [Access date Nov 26 2018].

11. Fung C, Pandya C, Guancial E, Noyes K, Sahasrabudhe DM, Messing EM, et al. Impact of bladder cancer on health related quality of life in 1,476 older Americans: a cross-sectional study. J Urol. 2014;192(3):690-5.

12. Goossens-Laan CA, Kil PJ, Ruud Bosch JL, De VJ. Pre-diagnosis quality of life $(\mathrm{QoL})$ in patients with hematuria: comparison of bladder cancer with other causes. Qual Life Res. 2013;22(2):309-15.
13. Perlis N, Krahn M, Alibhai S, Finelli A, Ritvo P, Bremner KE, et al. Conceptualizing global health-related quality of life in bladder cancer. Qual Life Res. 2014;23(8):2153-67.

14. Roychowdhury D, Hayden A, Liepa AM. Health-related quality-of-life parameters as independent prognostic factors in advanced or metastatic bladder cancer. J Clin Oncol. 2003:21(4):673-8.

15. von der MH, Hansen SW, Roberts JT, Dogliotti L, Oliver T, Moore MJ, et al. Gemcitabine and cisplatin versus methotrexate, vinblastine, doxorubicin, and cisplatin in advanced or metastatic bladder cancer: results of a large, randomized, multinational, multicenter, phase III study. J Clin Oncol. 2000;18(17):3068-77.

16. Joly F, Houede N, Noal S, Chevreau C, Priou F, Chinet-Charrot P, et al. Do patients with advanced urothelial carcinoma benefit from weekly paclitaxel chemotherapy? A GETUG phase II study. Clin Genitourin Cancer. 2009:7(2):E28-33.

17. De Santis M, Bellmunt J, Mead G, Kerst JM, Leahy M, Maroto P, et al. Randomized phase II/III trial assessing gemcitabine/carboplatin and methotrexate/carboplatin/vinblastine in patients with advanced urothelial cancer who are unfit for cisplatin-based chemotherapy: EORTC study 30986. J Clin Oncol. 2012;30(2):191-9.

18. Herman JM, Smith DC, Montie J, Hayman JA, Sullivan MA, Kent E, et al. Prospective quality-of-life assessment in patients receiving concurrent gemcitabine and radiotherapy as a bladder preservation strategy. Urology. 2004;64(1):69-73.

19. Albers $P$, Siener $R$, Hartlein M, Fallahi M, Haeutle D, Perabo FG, et al. Gemcitabine monotherapy as second-line treatment in cisplatin-refractory transitional cell carcinoma - prognostic factors for response and improvement of quality of life. Onkologie. 2002;25(1):47-52.

20. Mak KS, Smith AB, Eidelman A, Clayman R, Niemierko A, Cheng JS, et al. Quality of life in long-term survivors of muscle-invasive bladder Cancer. Int J Radiat Oncol Biol Phys. 2016;96(5):1028-36.

21. Hashine K, Miura N, Numata K, Shirato A, Sumiyoshi Y, Kataoka M. Healthrelated quality of life after bladder preservation therapy for muscle invasive bladder cancer. Int J Urol. 2008:15(5):403-6.

22. Huddart RA, Birtle A, Maynard L, Beresford M, Blazeby J, Donovan J, et al. Clinical and patient-reported outcomes of SP. BJU Int. 2017;120(5):639-50.

23. Henningsohn L, Wijkstrom H, Dickman PW, Bergmark K, Steineck G. Distressful symptoms after radical radiotherapy for urinary bladder cancer. Radiother Oncol. 2002;62(2):215-25.

24. Zietman AL, Sacco D, Skowronski U, Gomery P, Kaufman DS, Clark JA, et al. Organ conservation in invasive bladder cancer by transurethral resection, chemotherapy and radiation: results of a urodynamic and quality of life study on long-term survivors. J Urol. 2003;170(5):1772-6.

25. Allareddy V, Kennedy J, West MM, Konety BR. Quality of life in long-term survivors of bladder cancer. Cancer. 2006;106(11):2355-62.

26. Fokdal L, Hoyer M, Meldgaard P, von der MH. Long-term bladder, colorectal, and sexual functions after radical radiotherapy for urinary bladder cancer. Radiother Oncol. 2004;72(2):139-45

27. Lagrange $\mathrm{L}$, Bascoul-Mollevi C, Geoffrois L, Beckendorf V, Ferrero JM, Joly F et al. Quality of life assessment after concurrent chemoradiation for invasive bladder cancer: results of a multicenter prospective study (GETUG 97-015). Int J Radiat Oncol Biol Phys. 2011;79(1):172-8.

28. Seklehner S, Engelhardt PF, Remzi M, Fajkovic H, Saratlija-Novakovic Z, Skopek $\mathrm{M}$, et al. Anxiety and depression analyses of patients undergoing diagnostic cystoscopy. Qual Life Res. 2016;25(9):2307-14.

29. Lai C, Borrelli B, Ciurluini P, Aceto P. Sharing information about cancer with one's family is associated with improved quality of life. Psycho-Oncology. 2017;26(10):1569-75.

30. Rashid YA, Ghafoor ZA, Masood N, Mehmood T, Awan S, Ansar T, et al. Psychosocial impact of cancer on adult patients. J Pak Med Assoc. 2012;62(9):905-9.

31. Rottmann N, Dalton SO, Bidstrup PE, Wurtzen H, Hoybye MT, Ross L, et al. No improvement in distress and quality of life following psychosocial cancer rehabilitation. A randomised trial. Psycho-Oncology. 2012;21(5):505-14.

32. Walker LG, Kohler CR, Heys SD, Eremin O. Psychosocial aspects of cancer in the elderly. Eur J Surg Oncol. 1998;24(5):375-8.

33. Bellardita L, Villa S, Valdagni R. Living with untreated prostate cancer: predictors of quality of life. Curr Opin Urol. 2014;24(3):311-7.

34. Singer S, Ziegler C, Schwalenberg T, Hinz A, Gotze H, Schulte T. Quality of life in patients with muscle invasive and non-muscle invasive bladder cancer. Support Care Cancer. 2013;21(5):1383-93. 
35. Matsuda T, Aptel I, Exbrayat C, Grosclaude P. Determinants of quality of life of bladder cancer survivors five years after treatment in France. Int J Urol. 2003;10(8):423-9.

36. Mason SJ, Downing A, Wright P, Hounsome L, Bottomley SE, Corner J, et al. Health-related quality of life after treatment for bladder cancer in England. Br J Cancer. 2018;118(11):1518-28.

37. Butt Z, Rosenbloom SK, Abernethy AP, Beaumont IL, Paul D, Hampton $D$, et al. Fatigue is the most important symptom for advanced cancer patients who have had chemotherapy. J Natl Compr Cancer Netw. 2008;6(5):448-55.

38. Kent EE, Mitchell SA, Oakley-Girvan I, Arora NK. The importance of symptom surveillance during follow-up care of leukemia, bladder, and colorectal cancer survivors. Support Care Cancer. 2014;22(1):163-72

39. Henningsohn L, Wijkstrom H, Pedersen J, Ahlstrand C, Aus G, Bergmark K, et al. Time after surgery, symptoms and well-being in survivors of urinary bladder cancer. BJU Int. 2003;91(4):325-30

40. Gilbert SM, Wood DP, Dunn RL, Weizer AZ, Lee CT, Montie JE, et al. Measuring health-related quality of life outcomes in bladder cancer patients using the bladder Cancer index (BCl). Cancer. 2007;109(9):1756-62.

41. Krajewski W, Koscielska-Kasprzak K, Rymaszewska J, Zdrojowy R. How different cystoscopy methods influence patient sexual satisfaction, anxiety, and depression levels: a randomized prospective trial. Qual Life Res. 2017;26(3):625-34.

Ready to submit your research? Choose BMC and benefit from:

- fast, convenient online submission

- thorough peer review by experienced researchers in your field

- rapid publication on acceptance

- support for research data, including large and complex data types

- gold Open Access which fosters wider collaboration and increased citations

- maximum visibility for your research: over $100 \mathrm{M}$ website views per year

At BMC, research is always in progress.

Learn more biomedcentral.com/submissions 